\title{
Exponential synchronization of the high-dimensional Kuramoto model with identical oscillators under digraphs
}

\author{
Jinxing Zhang, Jiandong Zhu \\ Institute of Mathematics, School of Mathematical Sciences, Nanjing Normal University, Nanjing, 210023, PRC
}

\begin{abstract}
For the Kuramoto model and its variations, it is difficult to analyze the exponential synchronization under the general digraphs due to the lack of symmetry. In this paper, for the high-dimensional Kuramoto model of identical oscillators, a matrix Riccati differential equation (MRDE) is proposed to describe the error dynamics. Based on the MRDE, the exponential synchronization is proved by constructing a total error function for the case of digraphs admitting spanning trees. Finally, some numerical simulations are given to illustrate the obtained theoretical results.
\end{abstract}

Key words: High-dimensional Kuramoto model; Exponential synchronization; Directed graph.

\section{Introduction}

Kuramoto model is one of the most representative mathematical models of complex dynamical networks, which was first proposed by Yoshiki Kuramoto to describe and explain the synchronization phenomena in the real world [10]. Kuramoto model and its many variations have been applied to many fields such as neuro-science [3], power systems [5], chemical engineering [11], geophysics [22], and semiconductor lasers arrays [9]. The interconnecting network of the original Kuramoto model is a complete graph or the all-to-all topology. For general interconnecting topologies, the Kuramoto model composed of $m$ oscillators is described as

$$
\dot{\theta}_{i}=\omega_{i}+k \sum_{j=1}^{m} a_{i j} \sin \left(\theta_{j}-\theta_{i}\right), \quad i=1,2, \cdots, m,
$$

where $\theta_{i}$ is the phase of the $i$ th oscillator, $\omega_{i}$ is the natural frequency, $A=\left(a_{i j}\right)$ is the nonnegative adjacency matrix of the interconnection graph $\mathcal{G}$ and $k>0$ is the control gain.

In physics community, the researches pay more attentions on the thermodynamic limit described by a partial

\footnotetext{
* This work is supported in part by National Natural Science Foundation (NNSF) of China under Grants 61673012 and a project funded by the Priority Academic Program Development of Jiangsu Higher Education Institutions (PAPD).

Email addresses: jxingzhang@hotmail.com (Jinxing Zhang), zhujiandong@njnu.edu.cn (Jiandong Zhu).
}

differential equation as the number the oscillators tends to infinity. For details, we refer the reader to the surveys $[1,6]$. However, in system and control community, the main interest lies in the Kuramoto model composed of finite number of oscillators. Synchronization is a key issue on Kuromoto model and its variations. It is said that the phase synchronization is achieved if

$$
\lim _{t \rightarrow \infty}\left(\theta_{i}(t)-\theta_{j}(t)\right)=0 \quad \forall i, j=1,2, \cdots, m
$$

In [8], it is shown that, when $\mathcal{G}$ is an undirected graph, the Kuramoto model (1) can be rewritten as the compact form as follows:

$$
\dot{\theta}=\omega-k B \sin \left(B^{\mathrm{T}} \theta\right)
$$

where $\theta=\left(\theta_{1}, \theta_{2}, \cdots, \theta_{m}\right)^{\mathrm{T}}, \omega=\left(\omega_{1}, \omega_{2}, \cdots, \omega_{m}\right)^{\mathrm{T}}$, and $B$ is the incidence matrix of an oriented graph $\mathcal{G}^{\sigma}$ of $\mathcal{G}$. Just based on the compact form (2), the exponential phase synchronization is proved for the identical Kuramoto model with undirected graphs [8]. For the case of digraphs, the theoretical analysis of synchronization is relatively difficult. In [4] and [5], the contraction property is adopted to achieve the exponential synchronization for the Kuramoto model with digraphs.

An interesting issue is whether the synchronization or the exponential synchronization can be achieved for the high-dimensional Kuramoto model (Lohe model called 
in [2]) described as follows:

$$
\dot{r}_{i}=\Omega_{i} r_{i}+k \sum_{j=1}^{m} a_{i j}\left(r_{j}-\frac{r_{i}^{T} r_{j}}{r_{i}^{T} r_{i}} r_{i}\right), i=1,2, \cdots, m,
$$

where $r_{i} \in \mathbf{R}^{n}$ is the state of the $i$ th oscillator, $\Omega_{i}$ is an $n \times n$ skew-symmetric matrix, $k>0$ is the control gain, and $A=\left(a_{i j}\right) \in \mathbf{R}^{m \times m}$ is the weighted adjacency matrix of the interconnecting network. In [24], it has been shown that system (3) can be reduced to the original Kuramoto model (1) as $n=2$,

$$
\Omega_{i}=\left[\begin{array}{cc}
0 & -\omega_{i} \\
\omega_{i} & 0
\end{array}\right], r_{i}=\left[\begin{array}{c}
\cos \theta_{i} \\
\sin \theta_{i}
\end{array}\right] .
$$

The model (3) with $\Omega_{i}=0$ as well as the all-to-all interconnection is first proposed in [20] as a swarm model on spheres, and is used to solve the max-cut problem in combinatorial optimization. In $[13,14]$, some collective dynamical behaviors of (3) are shown, which have some potential applications in quantum synchronization of some quantum devises. For the case of complete graphs, exponential synchronization is proved by using the concept of order parameter in [2,7]. In [24],[25], [15] and [16], the phase synchronization on the unit hemisphere and the almost global synchronization are investigated for undirected graphs. For the case of digraphs, [12] and [23] achieve phase synchronization under some limitations on the initial states. In [17], a lifting method is proposed to analyze the almost globle synchronization on the unit sphere for digraphs. New exciting development on highdimensional Kuramoto model can be seen in $[18,19]$.

However, the theoretical analysis of the exponential synchronization is much more difficult for general graphs. By Theorem 13 of [16], the local exponential synchronization of (3) with $\Omega_{i}=0$ is achieved for connected undirected graphs by using the linearization method. From Theorem 1 of [12], one see that the exponential synchronization is implemented for weakly connected and balanced digraphs by using the invariant manifold techniques. Since a weakly connected and balanced digraph is strongly connected, the topology condition for the exponential synchronization imposed in [12] is very strong. To the best of the authors' knowledge, for general digraphs, the exponential synchronization problem of the high-dimensional Kuramoto model is still open.

In this paper, the exponential synchronization is proved for the high-dimensional Kuramoto model under a general digraph containing a spanning tree. A matrix Riccati differential equation is proposed to describe the dynamics of the synchronization errors for the first time, which plays an important role in the proof the exponential synchronization. Finally, numerical simulations are given to validate the obtained theoretical results.
The rest of this paper is organized as follows. Section 2 gives some preliminaries and the problem statement. Section 3 includes our main results. Section 4 shows some simulations. Finally, Section 5 is devoted to a summary.

\section{Preliminaries and Problem Statement}

Denote by $\mathcal{G}=(\mathcal{V}, \mathcal{E}, A)$ the weighted digraph of the high-dimensional Kuramoto model (3), which is composed a set of nodes $\mathcal{V}=\{1,2, \cdots, m\}$, set of edge $\mathcal{E} \subset \mathcal{V} \times \mathcal{V}$ and a weighted adjacent matrix $A=\left(a_{i j}\right)$ satisfying

$$
a_{i j} \begin{cases}>0, & (j, i) \in \mathcal{E} \\ =0, & \text { otherwise. }\end{cases}
$$

Here, a directed edge $(j, i)$ means that the state information of agent $j$ can be transmitted to $i$. A sequence of directed edges $\left(k_{1}, k_{2}\right),\left(k_{2}, k_{3}\right), \cdots,\left(k_{s-1}, k_{s}\right)$ is called a path from $k_{1}$ to $k_{s}$, denoted by $k_{1} k_{2} \cdots k_{s}$. If $\mathcal{G}$ has a node $v_{0}$ such that, for any another node $i$, there exists a directed path from $v_{0}$ to $i$, then $v_{0}$ is called a root node of $\mathcal{G}$. It is well-known that a digraph has a root node if and only if it has a directed spanning tree. The Laplacian matrix $L=\left(l_{i j}\right)$ of the weighted digraph $\mathcal{G}$ is defined by

$$
l_{i j}= \begin{cases}-a_{i j}, & i \neq j, \\ \sum_{k \neq i} a_{i k}, & i=j .\end{cases}
$$

Lemma 2.1 (Corollary 3 of [21]) Let $\mathcal{G}=(\mathcal{V}, \mathcal{E}, A)$ be a digraph with Laplacian matrix $L$. If $\mathcal{G}$ is strongly connected, then $L$ has a simple zero eigenvalue and a positive left-eigenvector associated to the zero eigenvalue.

Definition 2.1 For the high-dimensional Kuramoto model (3), the exponential synchronization is said to be implemented if there exist $\mu>0$ and a function $\alpha(r(0))$ with respect to the initial states of the oscillators such that

$$
\left\|r_{i}(t)-r_{j}(t)\right\| \leq \alpha(r(0)) \mathrm{e}^{-\mu t} \forall i, j=1,2, \cdots, m .
$$

For the high-dimensional Kuramoto model (3) with identical oscillators $\left(\Omega_{i}=\Omega\right.$ for each $\left.i=1,2, \cdots, m\right)$, without loss of generality, we can just consider the dynamics with each $r_{i}$ limited on the unit sphere as follows:

$$
\dot{r}_{i}=k \sum_{j=1}^{m} a_{i j}\left(r_{j}-\left(r_{i}^{T} r_{j}\right) r_{i}\right), i=1,2, \cdots, m .
$$

The first result on the synchronization of (6) with digraphs is Theorem 2 of [12], which is rewritten as follows by the terminologies in this paper.

Lemma 2.2 Assume that the digraph $\mathcal{G}$ of the highdimensional Kuromoto model (6) has a directed spanning 
tree and there exists $v \in \mathbf{R}^{n}$ such that $v^{\mathrm{T}} r_{i}(0)>0$ for every $i=1,2, \cdots, m$. Then there exists $\bar{r} \in \mathbf{R}^{n}$ such that $\lim _{t \rightarrow+\infty} r_{i}(t)=\bar{r}$ for each $i=1,2, \cdots, m$.

Remark 2.1 In Lemma 2.2, synchronization is implemented on the hemisphere. But in [17], a lifting method is proposed to analyze the global synchronization. In our recent paper [23], the synchronization of (6) in the case of digraphs is proved under some stronger conditions than those in Lemma 2.2 by using a completely different method.

\section{Exponential state synchronization}

In this section, we mainly consider the high-dimensional Kuramoto model (6) limited on the unit sphere.

Let

$$
e_{i j}=1-r_{i}^{T} r_{j}=\frac{1}{2}\left\|r_{i}-r_{j}\right\|^{2}
$$

It is easy to see that $e_{i j}=e_{j i}, e_{i i}=0$ and $0 \leq e_{i j} \leq 2$ for any $i, j=1,2, \cdots, m$. Obviously, $e_{i j}=0$ if and only if $r_{i}=r_{j}$. So $e_{i j}$ reflects the state error between $r_{i}$ and $r_{j}$. In the following, let us investigate the dynamics of all the $e_{i j}$ 's. A straightforward computation shows that

$$
\begin{aligned}
\dot{e}_{i j} & =-r_{j}^{\mathrm{T}} \dot{r}_{i}-r_{i}^{\mathrm{T}} \dot{r}_{j} \\
& =-k \sum_{l=1}^{m} a_{i l}\left(r_{j}^{\mathrm{T}} r_{l}-\left(r_{i}^{\mathrm{T}} r_{l}\right) r_{j}^{\mathrm{T}} r_{i}\right)-k \sum_{l=1}^{m} a_{j l}\left(r_{i}^{\mathrm{T}} r_{l}-\left(r_{j}^{\mathrm{T}} r_{l}\right) r_{i}^{\mathrm{T}} r_{j}\right) .
\end{aligned}
$$

Substituting $r_{i}^{T} r_{j}=1-e_{i j}$ into (8) yields

$$
\begin{aligned}
\dot{e}_{i j}= & k \sum_{l=1}^{m} a_{i l} e_{l j}-k\left(\sum_{l=1}^{m} a_{i l}\right) e_{i j}-k \sum_{l=1}^{m} a_{i l} e_{l i} \\
& +k\left(\sum_{l=1}^{m} a_{i l} e_{i l}\right) e_{i j}+k \sum_{l=1}^{m} a_{j l} e_{l i}-k\left(\sum_{l=1}^{m} a_{j l}\right) e_{i j} \\
& -k \sum_{l=1}^{m} a_{j l} e_{l j}+k\left(\sum_{l=1}^{m} a_{j l} e_{j l}\right) e_{i j}, i, j=1,2, \cdots, m
\end{aligned}
$$

Let $E=\left(e_{i j}\right) \in \mathbf{R}^{m \times m}$ and

$$
\alpha(E)=\left(\alpha_{1}(E), \alpha_{2}(E), \cdots, \alpha_{m}(E)\right)^{\mathrm{T}} \in \mathbf{R}^{m},
$$

where $\alpha_{i}(E)=\sum_{l=1}^{m} a_{i l} e_{i l}$. Denote by $\Lambda(E)=\operatorname{diag}(\alpha(E))$ the diagonal matrix with the main diagonal elements composed of $\alpha_{1}(E), \alpha_{2}(E), \cdots, \alpha_{m}(E)$. Then we can rewrite (9) into the compact form described by the Riccati matrix differential equation

$$
\begin{aligned}
\dot{E}= & -k L E-k E L^{\mathrm{T}}-k \alpha(E) \mathbf{1}^{\mathrm{T}}-k \mathbf{1} \alpha^{\mathrm{T}}(E) \\
& +k \Lambda(E) E+k E \Lambda(E),
\end{aligned}
$$

where $L$ is the Laplacian matrix of the digraph.

In the following, we will use (11) to investigate whether $E$ converges to zero exponentially. Before our main result on exponential synchronization, we first give a lemma.

Lemma 3.1 Consider a sequence of unit vectors $r_{1}, r_{2}, \cdots, r_{m} \in \mathbf{R}^{n}$. Let $e_{i j}$ be defined by (7) for any $i, j=1,2, \cdots m$. Then

$$
e_{i j} \leq s\left(e_{i k_{1}}+e_{k_{1} k_{2}}+\cdots+e_{k_{s-1, j}}\right)
$$

for any $s-1$ positive integers $k_{1}, k_{2}, \cdots, k_{s-1}$.

PROOF. Since $\left\|r_{i}-r_{j}\right\|^{2}=2 e_{i j}$, then we have

$$
\begin{aligned}
2 e_{i j} & =\left\|r_{i}-r_{j}\right\|^{2} \\
& \leq\left(\left\|r_{i}-r_{k_{1}}\right\|+\left\|r_{k_{1}}-r_{k_{2}}\right\|+\cdots+\left\|r_{k_{s-1}}-r_{j}\right\|\right)^{2} \\
& \leq s\left(\left\|r_{i}-r_{k_{1}}\right\|^{2}+\left\|r_{k_{1}}-r_{k_{2}}\right\|^{2}+\cdots+\left\|r_{k_{s-1}}-r_{j}\right\|^{2}\right) \\
& =2 s\left(e_{i k_{1}}+e_{k_{1} k_{2}}+\cdots+e_{k_{s-1}, j}\right)
\end{aligned}
$$

where the second inequality comes from the Cauchy inequality.

Lemma 3.2 Assume that the digraph $\mathcal{G}$ has a directed spanning tree with weighted adjacency matrix $A=\left(a_{i j}\right) \in \mathbf{R}^{m \times m}$. Then

(i) there exists a constant $c_{1}>0$ such that

$$
e_{i j} \leq c_{1} \sum_{p=1}^{m} \sum_{q=1}^{m} a_{p q} e_{p q}, \forall i, j=1,2, \cdots, m
$$

(ii) there exist constants $\check{c}>0$ and $\hat{c}>0$ such that

$$
\hat{c} \sum_{i=1}^{m} \sum_{j=1}^{m} e_{i j} \leq \sum_{p=1}^{m} \sum_{q=1}^{m} a_{p q} e_{p q} \leq \check{c} \sum_{i=1}^{m} \sum_{j=1}^{m} e_{i j}
$$

that is,

$$
\hat{c} \mathbf{1}_{m}^{\mathbf{T}} E \mathbf{1}_{m} \leq \mathbf{1}_{m}^{\mathbf{T}} \alpha(E) \leq \check{c} \mathbf{1}_{m}^{\mathbf{T}} E \mathbf{1}_{m}
$$

where $\alpha(E)$ is defined by (10).

PROOF. (i) Since $\mathcal{G}$ has a directed spanning tree, there exists a root node $v_{0}$ in $\mathcal{G}$. Then, for any $i \neq j$, there are two directed pathes $v_{0} k_{1} k_{2} \cdots k_{s} i$ and $v_{0} h_{1} h_{2} \cdots h_{t} j$. By Lemma 3.1, there exists a constant $c_{0}>0$

$e_{i j} \leq c_{0}\left(e_{v_{0} k_{1}}+e_{k_{1} k_{2}}+\cdots+e_{k_{s} i}+e_{v_{0} h_{1}}+e_{h_{1} h_{2}}+\cdots+e_{h_{t} j}\right)$

for all $i, j=1,2, \cdots, m$. Since all $a_{v_{0} k_{1}}, a_{k_{1} k_{2}}, \cdots, a_{k_{s} i}$, $a_{v_{0} h_{1}}, a_{h_{1} h_{2}}, \cdots, a_{h_{t} j}$ are positive, there is a constant 
$c_{1}>0$ such that

$$
\begin{aligned}
e_{i j} \leq & c_{1}\left(a_{v_{0} k_{1}} e_{v_{0} k_{1}}+a_{k_{1} k_{2}} e_{k_{1} k_{2}}+\cdots+a_{k_{s} i} e_{k_{s} i}\right) \\
& +c_{1}\left(a_{v_{0} h_{1}} e_{v_{0} h_{1}}+a_{h_{1} h_{2}} e_{h_{1} h_{2}}+\cdots+a_{h_{t} j} e_{h_{t} j}\right) \\
\leq & c_{1} \sum_{p=1}^{m} \sum_{q=1}^{m} a_{p q} e_{p q} .
\end{aligned}
$$

(ii) Let $\hat{c}=\frac{1}{c_{1} m^{2}}$ and $\check{c}=\max _{1 \leq p, q \leq m} a_{p q}$. Then (15) follows from (14).

\subsection{The case of strongly connected digraphs}

Theorem 3.1 Assume that the digraph $\mathcal{G}$ of the highdimensional Kuromoto model (6) is strongly connected and there exists $v \in \mathbf{R}^{n}$ such that $v^{\mathrm{T}} r_{i}(0)>0$ for every $i=1,2, \cdots, m$. Then the exponential synchronization of (6) is achieved.

PROOF. By Lemma 2.2, we see that the synchronization is achieved under the conditions of Theorem 3.1. In the rest of the proof, we use the error dynamics (11) to prove the exponential convergence. Since the digraph $\mathcal{G}$ is strongly connected, by Lemma 2.1 , there is a positive vector $\beta=\left(\beta_{1}, \cdots, \beta_{m}\right)^{\mathrm{T}} \in \mathbf{R}^{m}$ satisfying

$$
\beta^{\mathrm{T}} L=0, \quad \beta^{T} \mathbf{1}=1
$$

We construct a total error function as follows:

$$
V(E)=\frac{1}{2} \sum_{i=1}^{m} \sum_{j=1}^{m} \beta_{i} \beta_{j} e_{i j}=\frac{1}{2} \beta^{\mathrm{T}} E \beta .
$$

Let

$$
\Phi_{\eta}=\left\{E=\left(e_{i j}\right) \in \mathbf{R}^{m \times m} \mid e_{i j} \leq \eta, \forall i, j=1,2, \cdots, m\right\}
$$

and

$$
\Psi_{\eta}=\left\{E=\left(e_{i j}\right) \in \mathbf{R}^{m \times m} \mid V(E)<\hat{\beta}^{2} \eta / 2\right\},
$$

where $0<\eta<1$ and $\hat{\beta}=\min _{1 \leq i \leq m} \beta_{i}>0$.

Claim 1: $\Psi_{\eta} \subset \Phi_{\eta}$.

As a matter of fact, if $E \in \Psi_{\eta}$, then

$$
\frac{1}{2} \hat{\beta}^{2} e_{i j} \leq \frac{1}{2} \beta_{i} \beta_{j} e_{i j} \leq V(E)<\frac{1}{2} \hat{\beta}^{2} \eta
$$

which implies that $e_{i j}<\eta$. Thus Claim 1 is proved.
From (18), (11) and Claim 1, it follows that

$$
\begin{aligned}
\dot{V}(E) & =\frac{1}{2} \beta^{\mathrm{T}} \dot{E} \beta \\
& =-k \beta^{\mathrm{T}} \alpha(E)+k \beta^{T} \Lambda(E) E \beta \\
& =-k \sum_{i=1}^{m} \beta_{i} \sum_{l=1}^{m} a_{i l} e_{i l}+k \sum_{i=1}^{m} \sum_{j=1}^{m} \beta_{i}\left(\sum_{l=1}^{m} a_{i l} e_{i l}\right) e_{i j} \beta_{j} \\
& =-k \sum_{i=1}^{m} \sum_{l=1}^{m}\left(1-\sum_{j=1}^{m} \beta_{j} e_{i j}\right) \beta_{i} a_{i l} e_{i l} \\
& \leq-k(1-\eta) \hat{\beta} \sum_{i=1}^{m} \sum_{l=1}^{m} a_{i l} e_{i l}, \quad \forall E \in \Psi_{\eta} .
\end{aligned}
$$

Since the digraph $\mathcal{G}$ is strongly connected, by (19) and (15) of Lemma 3.2, we have

$$
\begin{aligned}
\dot{V}(E) & \leq-k(1-\eta) \hat{\beta} \hat{c} \sum_{i=1}^{m} \sum_{l=1}^{m} e_{i l}, \\
& \leq-k(1-\eta) \frac{\hat{\beta}}{\check{\beta}^{2}} \hat{c} \sum_{i=1}^{m} \sum_{l=1}^{m} \beta_{i} \beta_{l} e_{i l}, \\
& =-c V(E), \quad \forall E \in \Psi_{\eta},
\end{aligned}
$$

where $\check{\beta}=\max _{1 \leq i \leq m} \beta_{i}$ and $c=k(1-\eta) \frac{\hat{\beta}}{2 \dot{\beta}^{2}} \hat{c}>0$. By the definition of $\Psi_{\eta}$ and (20), we conclude that $\Psi_{\eta}$ is a positively invariant set with respect to $(11)$, and $V(E)$ converges to zero exponentially. In details, for any given initial states, there exists $T$ such that $e_{i j}(T) \in \Psi_{\eta}$ since the synchronization is achieved. By (20) and the positive invariance of $\Psi_{\eta}$, we have that

$$
V(E(t)) \leq V(E(T)) \mathrm{e}^{-c(t-T)}=\alpha_{1}(r(0)) \mathrm{e}^{-c t}, \forall t>T,
$$

where $\alpha_{1}(r(0))=V(E(T)) \mathrm{e}^{c T}$ is dependent on the initial states. Moreover, for the time interval $[0, T]$, there exists $\alpha_{2}(r(0))$ such that

$$
V(E(t)) \leq \alpha_{2}(r(0)) \leq \alpha_{2}(r(0)) \mathrm{e}^{c T} \mathrm{e}^{-c t}, \forall 0 \leq t \leq T
$$

From (21) and (22), it follows that there exists $\tilde{\alpha}(r(0))$ such that

$$
V(E(t)) \leq \tilde{\alpha}(r(0)) \mathrm{e}^{-c t}, \forall t \geq 0
$$

Further considering

$$
\left.\| r_{i}(t)-r_{j}(t)\right) \|^{2}=2 e_{i j}(t) \leq \frac{4}{\hat{\beta}^{2}} V(E(t)),
$$

we have

$$
\left.\| r_{i}(t)-r_{j}(t)\right) \| \leq \frac{2 \sqrt{\tilde{\alpha}(r(0))}}{\hat{\beta}} \mathrm{e}^{-\frac{c}{2} t}
$$


Therefore, by Definition 2.1, the exponential synchronization is achieved.

Remark 3.1 In the construction of the total error function $V(E)$, the strong connectedness of $\mathcal{G}$ plays an important role. From the proof of Theorem 3.1, we see that the crux of the analysis of exponential synchronization is to demonstrate $\dot{V}(E) \leq-c V(E)$ for some positive constant $c$ in a neighborhood of the origin $E=0$.

For the general case of digraphs admitting spanning trees, we will use all the strongly connected components to construct the total error function. For the readability of this paper, in the next subsection we assume that the considered digraph has only two strongly connected components.

3.2 The case of digraphs admitting two strongly connected components.

Proposition 3.1 Suppose that the digraph $\mathcal{G}$ of (6) has a spanning tree and is composed of two strongly connected components. Then the exponential synchronization is locally achieved, i.e., there exist a total error function $V(E)$ and a neighborhood $U$ of $E=0$ such that $\dot{V}(E) \leq-c V(E), \forall E \in U$ for some constant $c>0$.

PROOF. Let $\mathcal{G}_{1}=\left(\mathcal{V}_{1}, \mathcal{E}_{1}\right), \mathcal{G}_{2}=\left(\mathcal{V}_{2}, \mathcal{E}_{2}\right)$ be the two strongly connected components, where $\mathcal{V}_{1}=\{1,2, \cdots$, $\left.m_{1}\right\}, \mathcal{V}_{2}=\left\{m_{1}+1, m_{1}+2, \cdots, m_{1}+m_{2}\right\}$ and $m_{1}+m_{2}=m$. Without loss of generality, write the Laplacian matrix as

$$
L=\left[\begin{array}{cc}
L_{1} & 0 \\
-A_{21} & L_{2}+D_{2}
\end{array}\right]
$$

where $A_{21} \neq 0$ is a nonnegative matrix, $D_{2}$ is a diagnal nonegative matrix, $L_{1} \in \mathbf{R}^{m_{1} \times m_{1}}$ and $L_{2} \in \mathbf{R}^{m_{2} \times m_{2}}$ are the Laplacian matrices of $\mathcal{G}_{1}$ and $\mathcal{G}_{2}$, respectively. Consider the Lohe model (6) and the errors system (11). Let $E$ be partitioned as a 2 -by-2 block matrix

$$
E=\left[\begin{array}{ll}
E_{11} & E_{12} \\
E_{12}^{\mathrm{T}} & E_{22}
\end{array}\right]
$$

with $E_{11} \in \mathbf{R}^{m_{1} \times m_{1}}, E_{22} \in \mathbf{R}^{m_{2} \times m_{2}}$. Since both $\mathcal{G}_{1}$ and $\mathcal{G}_{2}$ are strongly connected, by Lemma 2.1 , there exist positive vectors $\beta_{1}=\left[\beta_{11}, \beta_{12}, \cdots, \beta_{1 m_{1}}\right]^{\mathrm{T}} \in \mathbf{R}^{m_{1}}$ and $\beta_{2}=\left[\beta_{21}, \beta_{22}, \cdots, \beta_{2 m_{2}}\right]^{\mathrm{T}} \in \mathbf{R}^{m_{2}}$ such that

$$
\beta_{1}^{\mathrm{T}} L_{1}=0, \quad \beta_{2}^{\mathrm{T}} L_{2}=0, \quad \beta_{1}^{\mathrm{T}} \mathbf{1}_{m_{1}}=\beta_{2}^{\mathrm{T}} \mathbf{1}_{m_{2}}=1 .
$$

Let $\beta=\left[\begin{array}{ll}\beta_{1}^{\mathrm{T}} & \varepsilon \beta_{2}^{\mathrm{T}}\end{array}\right]^{\mathrm{T}}$. Then $\beta^{\mathrm{T}} \mathbf{1}_{m}=1+\varepsilon$, where $\varepsilon>0$ is a sufficiently small constant. Construct the total error function as $V(E)=\frac{1}{2} \beta^{\mathrm{T}} E \beta$. Then a straightforward computation shows that

$$
\begin{aligned}
\dot{V}(E)= & -k \beta^{\mathrm{T}} L E \beta-k(1+\varepsilon) \beta^{\mathrm{T}} \alpha(E)+k \beta^{\mathrm{T}} \Lambda(E) E \beta \\
= & k \varepsilon \beta_{2}^{\mathrm{T}} A_{21} E_{11} \beta_{1}-k \varepsilon \beta_{2}^{\mathrm{T}} D_{2} E_{12}^{\mathrm{T}} \beta_{1} \\
& +k \varepsilon^{2} \beta_{2}^{\mathrm{T}} A_{21} E_{12} \beta_{2}-k \varepsilon^{2} \beta_{2}^{\mathrm{T}} D_{2} E_{22} \beta_{2} \\
& -k(1+\varepsilon)\left(\beta_{1}^{\mathrm{T}} \alpha_{1}(E)+\varepsilon \beta_{2}^{\mathrm{T}} \alpha_{2}(E)\right)+k \beta^{\mathrm{T}} \Lambda(E) E \beta \\
\leq & k \varepsilon \beta_{2}^{\mathrm{T}} A_{21} E_{11} \beta_{1}+k \varepsilon^{2} \beta_{2}^{\mathrm{T}} A_{21} E_{12} \beta_{2} \\
& -k \beta_{1}^{\mathrm{T}} \alpha_{1}(E)-k \varepsilon\left(\beta_{1}^{\mathrm{T}} \alpha_{1}(E)+\beta_{2}^{\mathrm{T}} \alpha_{2}(E)\right)+\gamma(E),
\end{aligned}
$$

where $\gamma(E)=k \beta^{\mathrm{T}} \Lambda(E) E \beta$ contains all the higher-order terms with respect to $E$. Letting

$$
\check{\beta}_{i}=\max _{1 \leq j \leq m_{i}} \beta_{i j}, \quad \hat{\beta}_{i}=\min _{1 \leq j \leq m_{i}} \beta_{i j}, \quad \check{a}=\max \left(A_{21}\right),
$$

by (27) and (26), we have

$$
\begin{aligned}
\dot{V}(E) \leq & \left(k \varepsilon \check{a} \check{\beta}_{1}\right) \mathbf{1}_{m_{1}}^{\mathrm{T}} E_{11} \mathbf{1}_{m_{1}}+\left(k \varepsilon^{2} \check{\beta}_{2} \check{a}\right) \mathbf{1}_{m_{1}}^{\mathrm{T}} E_{12} \mathbf{1}_{m_{2}} \\
& -\left(k \hat{\beta}_{1}\right) \mathbf{1}_{m_{1}}^{\mathrm{T}} \alpha_{1}(E)-\left(k \varepsilon \hat{\beta}_{1} \hat{\beta}_{2}\right) \mathbf{1}_{m}^{\mathrm{T}} \alpha(E)+\gamma(E) .
\end{aligned}
$$

Since $\mathcal{G}_{1}$ is strongly connected and $\mathcal{G}$ has a spanning tree, by (15) in Lemma 3.2 and (28), we have

$$
\begin{aligned}
\dot{V}(E) \leq & k\left(\varepsilon \check{\beta}_{2} \check{a} \check{\beta}_{1}-\hat{\beta}_{1} \hat{c}_{1}\right) \mathbf{1}_{m_{1}}^{\mathrm{T}} E_{11} \mathbf{1}_{m_{1}} \\
& +k \varepsilon\left(\varepsilon \check{\beta}_{2}^{2} \check{a}-\hat{\beta}_{1} \hat{\beta}_{2} \hat{c}\right) \mathbf{1}_{m}^{\mathrm{T}} E \mathbf{1}_{m}+\gamma(E),
\end{aligned}
$$

where $\hat{c}_{1}$ and $\hat{c}$ are determined by Lemma 3.2. By (29), as $\varepsilon$ is sufficiently small, there is a constant $\tilde{c}>0$ such that

$\dot{V}(E) \leq-\tilde{c} \mathbf{1}_{m}^{\mathrm{T}} E \mathbf{1}_{m}+\gamma(E)$

Since $\gamma(E)$ is composed of all the higher-order terms, there exists a neighborhood $U$ of $E=0$ such that

$\dot{V}(E) \leq-\frac{\tilde{c}}{2} \mathbf{1}_{m}^{\mathrm{T}} E \mathbf{1}_{m} \leq-\frac{\tilde{c}}{2 \hat{\beta}^{2}} \beta^{\mathrm{T}} E \beta=-c V(E), \quad \forall E \in U$,

where $c=\tilde{c} / \hat{\beta}^{2}>0$.

\subsection{The case of digraphs admitting spanning trees.}

Theorem 3.2 If the digraph $\mathcal{G}$ of the high-dimensional Kuramoto model (6) has a spanning tree, then the exponential synchronization is locally achieved.

PROOF. Denote all the strongly connected components of $\mathcal{G}$ by $\mathcal{G}_{1}, \mathcal{G}_{2}, \cdots, \mathcal{G}_{\mu}$, respectively. Let the number of the nodes of $\mathcal{G}_{i}$ be $m_{i}$ for each $i=1,2, \cdots, \mu$. 
Without loss of generality, assume that the Laplacian matrix $L$ has the form as follows:

$$
L=\left[\begin{array}{cccc}
L_{1} & & & \\
-A_{21} & L_{2}+D_{2} & & \\
\vdots & \ddots & \ddots & \\
-A_{\mu 1} & \cdots & -A_{\mu, \mu-1} & L_{\mu}+D_{\mu}
\end{array}\right],
$$

where $L_{i}$ is the Laplacian matrix of $\mathcal{G}_{i}$, each $A_{i j} \in$ $\mathbf{R}^{m_{i} \times m_{j}}$ is a nonnegative matrix, and every $D_{i} \in \mathbf{R}^{m_{i} \times m_{i}}$ is a nonnegative diagonal matrix. Corresponding to (31), we partition $E$ and $\alpha(E)$ as

$$
E=\left[\begin{array}{ccc}
E_{11} & \cdots & E_{1 \mu} \\
\vdots & \ddots & \vdots \\
E_{\mu 1} & \cdots & E_{\mu \mu}
\end{array}\right], \quad \alpha(E)=\left[\begin{array}{c}
\alpha_{1}(E) \\
\vdots \\
\alpha_{\mu}(E)
\end{array}\right],
$$

respectively, where $E_{i j}=E_{i j}^{\mathrm{T}} \in \mathbf{R}^{m_{i} \times m_{j}}$ for each $i, j=$ $1,2 \cdots, \mu$. Since $\mathcal{G}_{i}$ is strongly connected, by Lemma 2.1, there exists a positive vector $\beta_{i} \in \mathbf{R}^{m_{i}}$ such that

$$
\beta_{i}^{\mathrm{T}} L_{i}=0, \quad \beta_{i}^{\mathrm{T}} \mathbf{1}_{m_{i}}=1
$$

for each $i=1,2, \cdots, \mu$. Construct the total error function $V(E)=\frac{1}{2} \beta^{\mathrm{T}} E \beta$ with

$$
\beta=\left[\beta_{1}^{\mathrm{T}}, \quad \varepsilon \beta_{2}^{\mathrm{T}}, \quad \varepsilon^{2} \beta_{3}^{\mathrm{T}}, \cdots, \varepsilon^{\mu-1} \beta_{\mu}^{\mathrm{T}}\right]^{\mathrm{T}} .
$$

Then $\beta^{\mathrm{T}} \mathbf{1}_{m}=1+\varepsilon+\varepsilon^{2}+\cdots+\varepsilon^{\mu-1}$. A straightforward computation shows that

$$
\begin{aligned}
\dot{V}(E)= & -k \beta^{\mathrm{T}} L E \beta-k \beta^{\mathrm{T}} \alpha(E) \mathbf{1}_{m}^{\mathrm{T}} \beta+k \beta^{\mathrm{T}} \Lambda(E) E \beta \\
\leq & k \sum_{i=2}^{\mu} \sum_{j=1}^{i-1} \sum_{l=1}^{\mu} \varepsilon^{i+l-2} \beta_{i}^{\mathrm{T}} A_{i j} E_{j l} \beta_{l} \\
& -k \sum_{i=1}^{\mu} \varepsilon^{i-1} \sum_{j=1}^{\mu} \varepsilon^{j-1} \beta_{j}^{\mathrm{T}} \alpha_{j}(E)+\gamma(E),
\end{aligned}
$$

where $\gamma(E)=k \beta^{\mathrm{T}} \Lambda(E) E \beta$ contains all the higher-order terms with respect to $E$. Denote by $\tilde{\mathcal{G}}_{i}$ the subgraph composed of the first $i$ strongly connected components of $\mathcal{G}$. Let $\tilde{m}_{i}=m_{1}+m_{2}+\cdots+m_{i}$ and

$$
\tilde{E}_{i}=\left[\begin{array}{ccc}
E_{11} & \cdots & E_{1 i} \\
\vdots & \ddots & \vdots \\
E_{i 1} & \cdots & E_{i i}
\end{array}\right], \quad \tilde{\alpha}_{i}(E)=\left[\begin{array}{c}
\alpha_{1}(E) \\
\vdots \\
\alpha_{i}(E)
\end{array}\right]
$$

By the non-negativity of $A_{i j}$ and $\beta_{i}$, there exists a constant $d_{1}>0$ such that

$$
\beta_{i}^{\mathrm{T}} A_{i j} E_{j l} \beta_{l} \leq d_{1} \mathbf{1}_{m_{j}}^{\mathrm{T}} E_{j l} \mathbf{1}_{m_{i}} \leq d_{1} \mathbf{1}_{\tilde{m}_{j \vee l}}^{\mathrm{T}} \tilde{E}_{j \vee l} \mathbf{1}_{\tilde{m}_{j \vee l}},
$$

where $j \vee l=\max \{j, l\}$. Moreover, by (16) in Lemma 3.2 and the positivity of $\beta_{i}$ 's, it is easily seen that there exist positive constants $d_{2}$ and $d_{3}$ both independent of $\varepsilon$ such that

$$
\begin{aligned}
& \left(1+\varepsilon+\cdots+\varepsilon^{\mu-1}\right)\left(\beta_{1}^{\mathrm{T}} \alpha_{1}+\varepsilon \beta_{2}^{\mathrm{T}} \alpha_{2}+\cdots+\varepsilon^{\mu-1} \beta_{\mu}^{\mathrm{T}} \alpha_{\mu}\right) \\
\geq & \beta_{1}^{\mathrm{T}} \alpha_{1}+\varepsilon\left(\beta_{1}^{\mathrm{T}} \alpha_{1}+\beta_{2}^{\mathrm{T}} \alpha_{2}\right)+\cdots+\varepsilon^{\mu-1}\left(\beta_{1}^{\mathrm{T}} \alpha_{1}+\cdots+\beta_{\mu}^{\mathrm{T}} \alpha_{\mu}\right) \\
\geq & d_{2} \sum_{s=1}^{\mu} \varepsilon^{s-1} \mathbf{1}_{\tilde{m}_{s}}^{\mathrm{T}} \tilde{\alpha}_{s} \\
\geq & d_{3} \sum_{s=1}^{\mu} \varepsilon^{s-1} \mathbf{1}_{\tilde{m}_{s}}^{\mathrm{T}} \tilde{E}_{s} \mathbf{1}_{\tilde{m}_{s}} .
\end{aligned}
$$

Applying (37) and (38) to (35) yields

$$
\begin{aligned}
\dot{V}(E) \leq & k d_{1} \sum_{i=2}^{\mu} \sum_{j=1}^{i-1} \sum_{l=1}^{\mu} \varepsilon^{i+l-2} \mathbf{1}_{\tilde{m}_{j \vee l}}^{\mathrm{T}} \tilde{E}_{j \vee l} \mathbf{1}_{\tilde{m}_{j \vee l}} \\
& -k d_{3} \sum_{s=1}^{\mu} \varepsilon^{s-1} \mathbf{1}_{\tilde{m}_{s}}^{\mathrm{T}} \tilde{E}_{s} \mathbf{1}_{\tilde{m}_{s}}+\gamma(E) .
\end{aligned}
$$

From (39), we see that

$$
i+l-2 \geq i-1 \geq j, \quad i+l-2 \geq l .
$$

It follows from (40) that $i+l-2 \geq j \vee l$. So, by (39), we have

$$
\begin{aligned}
\dot{V}(E) \leq & -k \frac{d_{3}}{2} \sum_{s=1}^{\mu} \varepsilon^{s-1} \mathbf{1}_{\tilde{m}_{s}^{\mathrm{T}}}^{\mathrm{T}} \tilde{E}_{s} \mathbf{1}_{\tilde{m}_{s}}+\gamma(E) \\
& \leq-k \frac{d_{3}}{2} \varepsilon^{\mu-1} \mathbf{1}_{m}^{\mathrm{T}} E \mathbf{1}_{m}+\gamma(E)
\end{aligned}
$$

for sufficiently small $\varepsilon>0$. So there exist a constant $c>0$ and a neighborhood $U$ of the origin $E=0$ such that $\dot{V}(E) \leq-c V(E)$, which implies that $V(E)$ converges to zero exponentially.

Combining Lemma 2.2 and Theorem 3.2 yields the following result:

Theorem 3.3 Assume that the digraph $\mathcal{G}$ of the highdimensional Kuromoto model (6) has a directed spanning tree and there exists $v \in \mathbf{R}^{n}$ such that $v^{\mathrm{T}} r_{i}(0)>0$ for every $i=1,2, \cdots, m$. Then the exponential synchronization of (6) is achieved.

Assume that the high-dimensional Kuramoto model with identical oscillators has the general form as follows

$$
\dot{r}_{i}=\Omega r_{i}+k \sum_{j=1}^{m} a_{i j}\left(r_{j}-\left(r_{i}^{T} r_{j}\right) r_{i}\right), i=1,2, \cdots, m
$$




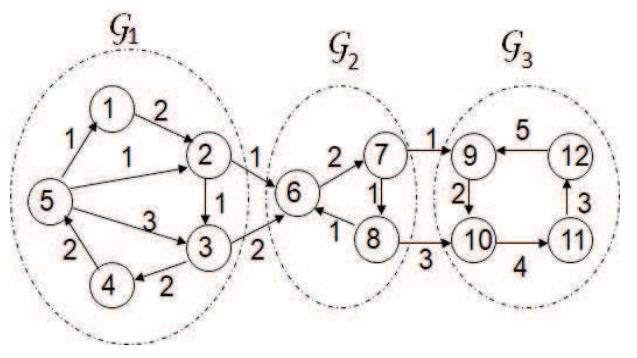

Fig. 1. The digraph of the high-dimensional Kuramoto model.

where $\Omega$ is a skew-symmetric matrix. Then, by [24], a system transformation can be adopted to transform system (42) to the form of (6). Therefore, the conclusion of Theorem 3.3 still holds for system (42).

\section{Simulations}

In this section, we give some simulations to show the exponential synchronization. Consider the highdimensional Kuramoto model (6) with $n=3$ and $m=12$. The digraph is shown in Fig. 1, which has 3 strongly connected components. From Fig. 1, we obtain the adjacency matrix as follows:

$$
A=\left(a_{i j}\right)=\left[\begin{array}{llllllllllll}
0 & 0 & 0 & 0 & 1 & 0 & 0 & 0 & 0 & 0 & 0 & 0 \\
2 & 0 & 0 & 0 & 1 & 0 & 0 & 0 & 0 & 0 & 0 & 0 \\
0 & 1 & 0 & 0 & 3 & 0 & 0 & 0 & 0 & 0 & 0 & 0 \\
0 & 0 & 2 & 0 & 0 & 0 & 0 & 0 & 0 & 0 & 0 & 0 \\
0 & 0 & 0 & 2 & 0 & 0 & 0 & 0 & 0 & 0 & 0 & 0 \\
0 & 1 & 2 & 0 & 0 & 0 & 0 & 1 & 0 & 0 & 0 & 0 \\
0 & 0 & 0 & 0 & 0 & 2 & 0 & 0 & 0 & 0 & 0 & 0 \\
0 & 0 & 0 & 0 & 0 & 0 & 1 & 0 & 0 & 0 & 0 & 0 \\
0 & 0 & 0 & 0 & 0 & 0 & 1 & 0 & 0 & 0 & 0 & 5 \\
0 & 0 & 0 & 0 & 0 & 0 & 0 & 3 & 2 & 0 & 0 & 0 \\
0 & 0 & 0 & 0 & 0 & 0 & 0 & 0 & 0 & 4 & 0 & 0 \\
0 & 0 & 0 & 0 & 0 & 0 & 0 & 0 & 0 & 0 & 3 & 0
\end{array}\right] .
$$

Then, the dynamics with each $r_{i}$ limited on the unit sphere is

$$
\dot{r}_{i}=k \sum_{j=1}^{12} a_{i j}\left(r_{j}-\left(r_{i}^{T} r_{j}\right) r_{i}\right), i=1,2, \cdots, 12 .
$$

From Fig. 1, the Laplacian matrices of $\mathcal{G}_{1}, \mathcal{G}_{2}$ and $\mathcal{G}_{3}$ are

$$
L_{1}=\left[\begin{array}{ccccc}
1 & 0 & 0 & 0 & -1 \\
-2 & 3 & 0 & 0 & -1 \\
0 & -1 & 4 & 0 & -3 \\
0 & 0 & -2 & 2 & 0 \\
0 & 0 & 0 & -2 & 2
\end{array}\right], L_{2}=\left[\begin{array}{ccc}
1 & 0 & -1 \\
-2 & 2 & 0 \\
0 & -1 & 1
\end{array}\right], L_{3}=\left[\begin{array}{cccc}
5 & 0 & 0 & -5 \\
-2 & 2 & 0 & 0 \\
0 & -4 & 4 & 0 \\
0 & 0 & -3 & 3
\end{array}\right],
$$

respectively. A straightforward computation shows that

$$
\beta_{1}^{\mathrm{T}}=[0.1111,0.0556,0.1667,0.3333,0.3333],
$$
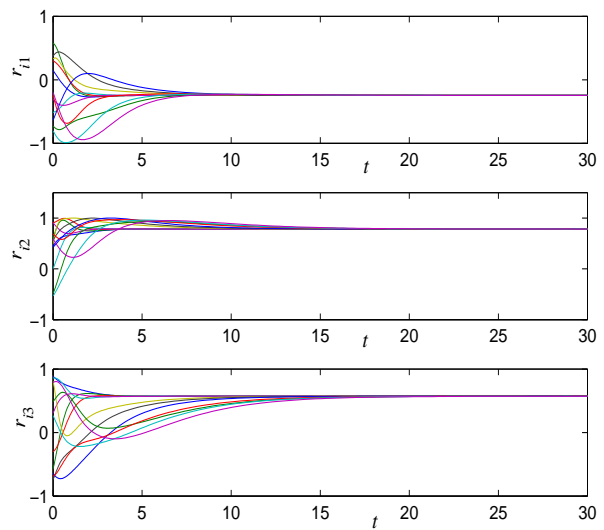

Fig. 2. The time response curves as $k_{i}=1$.

$\beta_{2}^{\mathrm{T}}=[0.4,0.2,0.4], \beta_{3}^{\mathrm{T}}=[0.1558,0.3896,0.1948,0.2597]$.

Construct the total error function $V(E)=\frac{1}{2} \beta^{\mathrm{T}} E \beta$ with

$$
\beta=\left[\begin{array}{lll}
\beta_{1}^{\mathrm{T}}, & \varepsilon \beta_{2}^{\mathrm{T}}, & \varepsilon^{2} \beta_{3}^{\mathrm{T}}
\end{array}\right]^{\mathrm{T}} .
$$

Fig.2 shows that the time response curves of agent's are synchronized. Fig.3 shows that all the trajectories of the oscillators converge to the same point on the unit sphere.

Consider the high-dimensional Kuramoto model with the form as follows:

$$
\dot{r}_{i}=\Omega r_{i}+k \sum_{j=1}^{12} a_{i j}\left(r_{j}-\left(r_{i}^{T} r_{j}\right) r_{i}\right), i=1,2, \cdots, 12,
$$

where

$$
\Omega=\left[\begin{array}{ccc}
0 & 1 & -2 \\
-1 & 0 & -1 \\
2 & 1 & 0
\end{array}\right]
$$

Fig.4 shows that the dynamical synchronization is achieved. Fig.5 shows that the trajectories converge to a periodic orbit. Finally, we display the total error function $V$. In Fig.6, it is shown that the total error function tends to zero exponentially as $t \rightarrow \infty$.

\section{Conclusions}

The exponential synchronization has been proved for the high-dimensional Kuramoto model with identical oscillators under the digraphs admitting spanning trees. The error dynamics is described by a matrix Riccati differential equation and a total error function is constructed for the analysis of the exponential synchronization. In our future work, the exponential synchronization will be investigate for some generalized highdimensional Kuramoto model. For the high-dimensional 


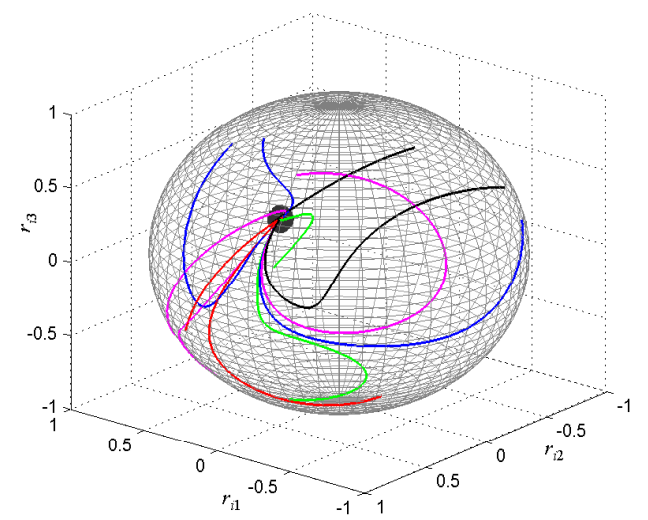

Fig. 3. The trajectories of the oscillators as $k_{i}=1$.
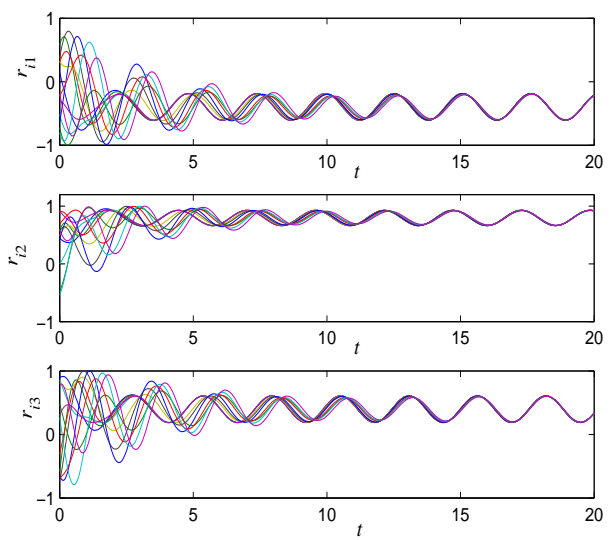

Fig. 4. The time response curves as $k_{i}=1$.

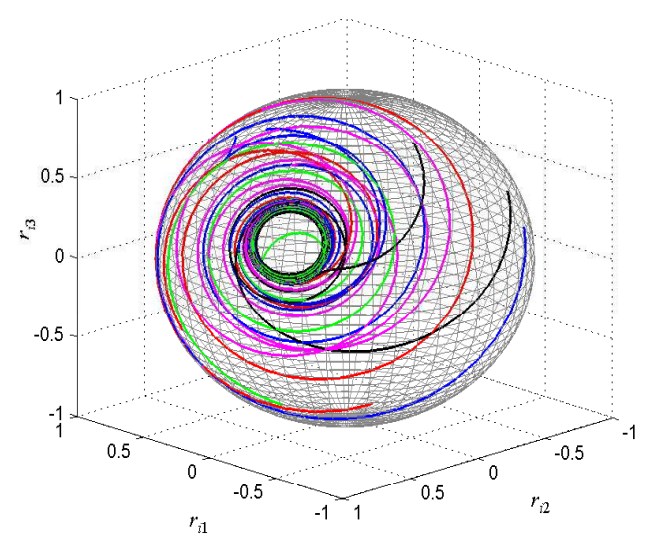

Fig. 5. The trajectories of the oscillators as $k_{i}=1$.

Kuramoto model with non-identical oscillators, practical synchronization will be addressed. How to generalize the frequency synchronization into the high-dimensional spaces is also an interesting issue.

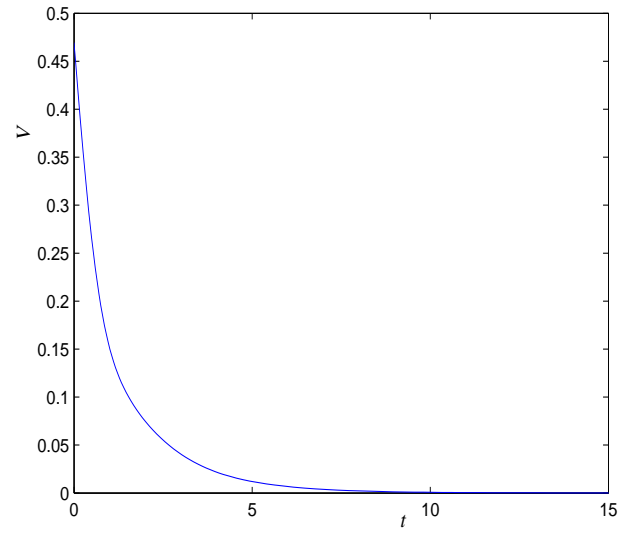

Fig. 6. The time response curves of the total error function.

\section{References}

[1] Acebrón, J. A., Bonilla, L. L., Vicente, C. J. P., Ritort, F., \& Spigler, R. (2005). The Kuramoto model: a simple paradigm for synchronization phenomena. Reviews of Modern Physics, $77(1), 137-185$.

[2] Choi, S.-H., \& Ha, S.-Y. (2014). Complete entrainment of lohe oscillators under attractive and repulsive couplings. SIAM Journal on Applied Dynamical Systems, 13(4), 1417-1441.

[3] Cumin, D., \& Unsworth, C. P. (2007). Generalising the Kuramoto model for the study of neuronal synchronisation in the brain. Physica D: Nonlinear Phenomena, 226(2), 181196.

[4] Dong, J.-G., \& Xue, X. (2013). Synchronization analysis of Kuramoto oscillators. Communications in Mathematical Sciences, 11(2), 465-480.

[5] Dörfler, F., \& Bullo, F. (2012). Synchronization and transient stability in power networks and nonuniform Kuramoto oscillators. SIAM Journal on Control and Optimization, 50(3), 1616-1642.

[6] Dörfler, F., \& Bullo, F. (2014). Synchronization in complex networks of phase oscillators: A survey. Automatica, 50(6), 1539-1564.

[7] Chi, D., Choi, S. H., \& Ha, S. Y. (2014). Emergent behaviors of a holonomic particle system on a sphere. Journal of Mathematical Physics, 55(5), 052703.

[8] Jadbabaie, A., Motee, N. \& Barahona, M. (2004). On the stability of the Kuramoto model of coupled nonlinear oscillators. In Proceeding of the 2004 american control conference,(pp. 4296-4301). Boston, Massachusetts, USA.

[9] Kozyreff, G., Vladimirov, A. G., \& Mandel, P. (2000). Global coupling with time delay in an array of semiconductor lasers. Physical Review Letters, 85(18), 3809-3812.

[10] Kuramoto, Y. (1975). Self-entrainment of a population of coupled non-linear oscillators. In Proceedings of international symposium on mathematical problems in theoretical physics, lecture notes in physics, 39,(pp. 420-422). Kyoto, Japan.

[11] Kuramoto, Y. (1984). Chemical oscillations, waves, and turbulence. Berlin: Springer-Verlag.

[12] Lageman, C., \& Sun Z. Y. (2016). Consensus on spheres: convergence analysis and perturbation theory. In Proceedings of the 55th IEEE conference on decision and control, (pp. 19-24). Las Vegas, Nevada, USA. 
[13] Lohe, M. A. (2009). Non-Abelian Kuramoto model and synchronization. Journal of Physics A: Mathematical and Theoretical, 42(39), 395101.

[14] Lohe, M. A. (2010). Quantum synchronization over quantum networks. Journal of Physics A Mathematical \& Theoretical, 43(46), 465301.

[15] Markdahl, J., \& Gonçalves, J. (2016). Global converegence properties of a consensus protocol on the $n$-Sphere. In Proceedings of the 55th IEEE conference on decision and control, (pp. 3487-3492). Las Vegas, Nevada, USA.

[16] Markdahl, J., Thunberg, J., \& Gonçalves, J. (2018). Almost Global Consensus on the $n$-Sphere. IEEE Transactions on Automatic Control, 63(6), 1664-1675.

[17] Thunberg, J., Markdahl, J., Bernard, F., \& Goncalves, J. (2018). A lifting method for analyzing distributed synchronization on the unit sphere. Automatica. (Accepted) https://arxiv.org/abs/1805.02528

[18] Markdahl, J. Thunberg, J., \& Goncalves, J. (2018). High-dimenatsional Kuramoto models on Stiefel manifolds synchronize complex networks almost globally. arXiv preprint arXiv:1807.10233

[19] Markdahl, J. (2018). A Topological obstruction to almost global synchronization on Riemannian manifolds. arXiv preprint arXiv:1808.00862

[20] Olfati-Saber, R. (2006). Swarms on sphere: A programmable swarm with synchronous behaviors like oscillator networks. In Proceedings of the 45th IEEE conference on decision and control (pp. 5060-5066). San Diego, California, USA.

[21] Scutari, G., Barbarossa, S., \& Pescosolido, L. (2008). Distributed decision through self synchronizing sensor networks in the presence of propagation delays and asymmetric channels. IEEE Transactions on Signal Processing, 56(4), 1667-1684.

[22] Vasudevan, K., Cavers, M., \& Ware, A. (2015). Earthquake Sequencing: Chimera States with Kuramoto Model Dynamics on Directed Graphs. Nonlinear Processes in Geophysics, 22(1), 499-512.

[23] Zhang, J., Zhu, J., \& Qian, C. (2018). On equilibria and consensus of Lohe model with identical oscillators. SIAM Journal on Applied Dynamical Systems, 17(2), 1716-1741.

[24] Zhu, J. (2013). Synchronization of Kuramoto model in a high-dimensional linear space. Physics Letters A, 377(41), 2939-2943.

[25] Zhu, J. (2014). High-dimensional Kuramoto model limited on smooth curved surfaces. Physics Letters A, 378(18-19), 1269-1280. 